Ambiente \& Água - An Interdisciplinary Journal of Applied Science
ISSN 1980-993X - doi:10.4136/1980-993X
www.ambi-agua.net
E-mail: ambi.agua@gmail.com

\title{
Modelación matemática del transporte de oxadixyl en suelos de cultivo de cebolla
}

\author{
doi: 10.4136/ambi-agua.1565 \\ Received: 07 Nov. 2014; Accepted: 18 Feb. 2015
María José Martínez Cordón ; Marta Isabel Aldana Castañeda; Jairo Arturo Guerrero Dallos \\ Universidad Nacional de Colombia (UNAL), Bogotá, Cundinamarca, Colombia \\ Departamento de Química \\ *Autor correspondiente: e-mail: mjmartinezc@unal.edu.co, \\ mialdanac@unal.edu.co,jaguerrerod@unal.edu.co
}

\section{RESUMEN}

Los plaguicidas utilizados en la producción de cultivos son la fuente más importante de contaminación difusa hacia las aguas subterráneas, y su descarga en la superficie del agua puede ser un factor que contribuye a la disminución de los recursos vivos y el deterioro de los ecosistemas. En este trabajo se estudió el movimiento de oxadixyl a través de columnas de suelo (30 cm de longitud y $14 \mathrm{~cm}$ de diámetro) en condiciones de laboratorio utilizando un suelo de cultivo de cebolla del lago de Tota (Boyacá, Colombia). Se inyectó un pulso de solución de $\mathrm{CaCl}_{2}$ 0,01 M, que contenía un trazador (bromuro) y oxadixyl en la superficie del suelo de la columna, a continuación, se aplicó lluvia simulada a una intensidad de $0,034 \mathrm{~cm}$ $\mathrm{h}^{1}$. Después de 2,13 volúmenes relativos de poro, en 30 días, el porcentaje de oxadixyl recuperado en la parte inferior de la columna fue de $92,1 \%$. La curva experimental de elución de oxadixyl se analizó utilizando el programa Stanmod (problema inverso) para obtener los parámetros de transporte. El modelo de no equilibrio químico describió bien las curvas de elución experimentales. En particular, la cola de elución fue bien ajustada. El factor de retardo calculado para el fungicida fue de 3,94 y el coeficiente de partición, $\mathrm{Kd}$, fue cercano a $1 \mathrm{Kg} \mathrm{L}^{1}$, lo que indica una baja adsorción en este suelo. Bajo nuestras condiciones experimentales, oxadixyl es móvil en este suelo y presenta un riesgo potencial de contaminación de las aguas subterráneas.

Palabras clave: columnas de suelo, curvas de elución, fungicidas, programa Stanmod, retención.

\section{Modelagem matemática de transporte de oxadixil em solo de cultivo de cebola}

\section{RESUMO}

Os praguicidas utilizados na produção de cultivos são a fonte mais importante de contaminação difusa das águas subterrâneas, e sua descarga na superficie da água pode ser um fator que contribui para a diminuição dos recursos vivos e deterioração dos ecossistemas. $\mathrm{O}$ movimento de oxadixyl através de colunas de solo $(30 \mathrm{~cm}$ y $14 \mathrm{~cm}$ de diametro) foi estudado em condições de laboratório utilizando um solo de cultivo de cebola do Lago de Tota 
(Boyacá, Colombia). Foi colocada uma solução de $\mathrm{CaCl}_{2}$ 0,01 M, contento um marcador (brometo) e oxadixil na superficie do solo, em seguida, aplicou-se chuva simulada a una intensidad de $0,034 \mathrm{~cm} \mathrm{~h}^{-1}$. Depois de 2,13 volumes de poro, em 30 días, a porcentagem de oxadixil recuperado na parte inferior da coluna foi de 92,1\%. Analisou-se a curva experimental de eluição de oxadixyl utilizando-se o programa Stanmod (problema inverso) para obter os parâmetros de transporte. O modelo de não-equilíbrio químico descreve bem as curvas de eluição experimentais. Em particular, a cauda de eluição foi bem ajustada. $O$ fator de retardamento calculado para o fungicida foi de 3,94 e o coeficiente de partição foi próximo de $1 \mathrm{~kg} \mathrm{~L}^{-1}$, o que indica baixa absorção deste solo. Sob as condições experimentais, oxadixyl (fase móvel) neste solo apresenta um risco potencial de contaminação das águas subterrâneas.

Palavras-chave: colunas de solo, curva de eluição, fungicida, programa Stanmod, retenção.

\title{
Mathematical modeling of oxadixyl transport in onion crop soil
}

\begin{abstract}
Pesticides used in crop production are the most important source of diffuse pollution to groundwater, and their discharge into surface water may be a contributing factor towards the decline of living resources and the deterioration of ecosystems. In this work, we studied the movement of oxadixyl through soil columns (30 cm length and $14 \mathrm{~cm}$ diameter) in laboratory conditions using onion soil from Lake Tota (Boyacá, Colombia). A solution of $0.01 \mathrm{M} \mathrm{CaCl}_{2}$, containing a tracer (bromide) and oxadixyl was sprayed onto the surface of the soil column, and then simulated rainfall was applied at an intensity of $0.034 \mathrm{~cm} \mathrm{~h}^{-1}$. After 30 days, and 2.13 relative pore volumes, oxadixyl percentages recovered at the bottom of the column were $92.1 \%$. The oxadixyl experimental elution curve was analyzed using the Stanmod program (inverse problem) to obtain transport parameters. The non-equilibrium chemical model described the experimental elution curve well. The tail of the elution curve was particularly well captured. The retardation factor calculated for the fungicide was 3.94 and the partition coefficient, $\mathrm{kd}$, was close to $1 \mathrm{~kg} \mathrm{~L}^{-1}$, indicating low adsorption in this soil. Under the experimental conditions, it could be concluded that oxadixyl is mobile in this soil, and therefore presents a risk of potential groundwater contamination.
\end{abstract}

Keywords: breakthrough curve, fungicides, retention, soil columns, Stanmod program.

\section{INTRODUCCIÓN}

La Cuenca hidrográfica del Lago de Tota está situada en la región centro oriental del departamento de Boyacá, Colombia $\left(05^{\circ} 32^{\prime} \mathrm{N}, 72^{\circ} 56^{\prime} \mathrm{E}\right)$ a 3.015 metros sobre el nivel del mar, y cubre un área de $6000 \mathrm{Ha}$ con un perímetro de $55 \mathrm{Km}$, convirtiéndose en el lago más grande de Colombia. Es el mayor abastecedor de agua del valle de Sogamoso, siendo utilizado por más de 500.000 habitantes de los municipios aledaños como fuente de agua para consumo humano, agrícola e industrial (Molina, 2007). La principal actividad agrícola de la zona es el cultivo de la cebolla larga (Allium fistulosum), que representa aproximadamente el $70 \%$ de la producción nacional (Pinzón, 2004). Las condiciones generales de producción son el cultivo intensivo, que incluyen, riego por aspersión, labranza del suelo, aplicación de elevadas dosis de fertilizantes y empleo excesivo de plaguicidas para prevenir enfermedades y plagas de la cebolla, que se ven favorecidas por las altas precipitaciones y la incorporación de las enmiendas orgánicas (Herrera et al., 2006; Mójica, 2010).

Los recursos hídricos de la región están amenazados por dos principales fuentes de contaminación, los vertimientos domésticos y los agroquímicos (Mójica, 2010; Abella y Martínez, 2012). Los insumos agrícolas una vez aplicados se pueden transportar hacia las 
aguas superficiales, especialmente los plaguicidas que provienen de las frecuentes fumigaciones que se realizan para preservar la producción de cebolla. Las dosis y frecuencias de uso de los plaguicidas son establecidas de acuerdo a la agresividad de las enfermedades, encontrándose que los agricultores aplican cada 8 o 15 días (Mójica, 2010).

El oxadixyl, 2-methoxy-N-(2-oxo-1,3-oxazolidin-3-yl)acet-2',6'-xylidida, es un fungicida de categoría toxicológica III registrado para varios cultivos: papa, rosas, tomate cebolla bulbo, tomate de arbol, lulo, berenjena, cebolla larga puerro (Ica, 2014) y empleado en la zona de estudio. Su solubilidad en agua es $3400 \mathrm{mg} \mathrm{L}^{-1}$, y posee un coeficiente de partición en la materia orgánica (Koc) en el rango de 24-56 L kg-1 (Iupac, 2014). En la bibliografía se han descrito tiempos de vida media, $\mathrm{DT}_{50}$, comprendidos entre 75-225 días. Debido a su baja tendencia a adsorberse en materia orgánica, oxadixyl puede ser un potencial contaminante de aguas superficiales (Saiano y Ciofalo, 2007; Iupac, 2014), y como consecuencia de su alta solubilidad en agua y alta movilidad, puede llegar hasta aguas subterráneas y contaminar el agua utilizada para consumo humano en la región. Sin embargo no se han encontrado estudios específicos sobre la lixiviación de oxadixyl, ni en condiciones de campo ni de laboratorio, aunque en estudios realizados en Francia, oxadixyl ha sido detectado en el 26\% de las aguas superficiales monitoreadas (Blanchoud et al., 2007).

Por ello el objetivo de este trabajo fue evaluar el transporte de oxadixyl a través del perfil del suelo de un cultivo de cebolla situado en la cuenca del Lago de Tota, empleando una columna de suelo en condiciones de laboratorio y aplicando un modelo matemático de transporte que nos permita caracterizar los parámetros de retención para el xenobiótico.

\section{MATERIALES Y MÉTODOS}

\subsection{Reactivos}

Se empleó estándar analítico de oxadixyl de pureza 98,5\% (Dr. Ehrenstorfer) y $\mathrm{KBr}$ 99,8\% (Merck). El acetonitrilo grado HPLC, se obtuvo de J.T. Baker y el agua grado HPLC fue obtenida por el sistema Milli/Q.

\subsection{Toma de muestras, caracterización y columna de suelo}

El suelo fue recolectado a diferentes profundidades: 0-10, 10-20 y 20-30 cm en un cultivo de cebolla larga situado en Aquitania (Boyacá, Colombia). Las muestras fueron secadas al aire, tamizadas $\left(2 \mathrm{~mm}\right.$, tamiz ASTM n $\left.{ }^{\circ} 10\right)$ y almacenadas a $4^{\circ} \mathrm{C}$ hasta el momento de los análisis. Se evaluaron los siguientes parámetros fisicoquímicos del suelo: Densidad aparente, Textura, Materia Orgánica (MO), pH y Capacidad de Intercambio Catiónico (CIC). Las propiedades fisicoquímicas del suelo, a las tres profundidades, se presentan en la Tabla 1. Cada análisis fue realizado por triplicado y el resultado se expresó como el promedio de las tres determinaciones.

El suelo se empacó manualmente respetando las tres profundidades, en una columna de PVC de $30 \mathrm{~cm}$ de longitud y $14 \mathrm{~cm}$ de diámetro interno. La columna se sometió a vibración por etapas, hasta obtener la densidad aparente correspondiente a cada profundidad (Tabla 1). Previo al inicio de los ensayos de infiltración el suelo se humedeció por capilaridad con una solución de $\mathrm{CaCl}_{2}$ 0,01 M, con el fin de evitar la presencia de burbujas de aire y tener una humedad uniforme.

\subsection{Ensayo de infiltración}

Los ensayos de infiltración se realizaron bajo condiciones de flujo constante y no saturación. Para mantener condiciones de insaturación se aplicó una presión negativa por diferencia de altura entre la base de la columna y el nivel constante de agua a la salida de la columna $(10 \mathrm{~cm})$ mientras la superficie del suelo se mantuvo bajo condiciones de presión 
atmosférica. Se ajustó el flujo, hasta que se mantuvo constante, mediante el uso de una bomba persitáltica y un simulador de lluvia compuesto por 45 orificios equidistantes. Durante el ensayo el dispositivo se mantuvo a una temperatura de entre $18-20^{\circ} \mathrm{C}$ y oscuridad para evitar fenómenos de fotodescomposición del plaguicida.

Tabla 1. Propiedades fisicoquímicas del suelo de estudio a tres profundidades: $0-10 \mathrm{~cm}, 10-20 \mathrm{~cm}$ y $20-30 \mathrm{~cm}$.

\begin{tabular}{|c|c|c|c|c|}
\hline \multirow{2}{*}{\multicolumn{2}{|c|}{ Propiedades }} & \multicolumn{3}{|c|}{ Profundidad cm } \\
\hline & & $0-10$ & 0-20 & 0-30 \\
\hline \multirow{3}{*}{ Textura } & $\%$ Arcillas & $33 \pm 1$ & $42 \pm 1$ & $45 \pm 1$ \\
\hline & $\%$ Arenas & $29 \pm 1$ & $47 \pm 1$ & $35 \pm 0$ \\
\hline & $\%$ Limos & $38 \pm 1$ & $11 \pm 2$ & $20 \pm 1$ \\
\hline \multicolumn{2}{|c|}{ Clase textural } & Franco Arcilloso & Arcilloso & Arcilloso \\
\hline \multicolumn{2}{|l|}{$\%$ MO } & $5,57 \pm 0,22$ & $5,23 \pm 0,32$ & $4,73 \pm 0,32$ \\
\hline \multicolumn{2}{|l|}{ pH } & $5,44 \pm 0,01$ & $5,42 \pm 0,02$ & $5,43 \pm 0,01$ \\
\hline \multicolumn{2}{|c|}{ Capacidad intercambio catiónico (Meq $100 \mathrm{~g}^{-1}$ ) } & $32,26 \pm 0,44$ & $27,94 \pm 0,32$ & $13,17 \pm 0,55$ \\
\hline \multicolumn{2}{|c|}{ Densidad aparente $\mathrm{g} \mathrm{cm}^{-3}$} & $1,37 \pm 0,11$ & $1,32 \pm 0,07$ & $1,31 \pm 0,06$ \\
\hline
\end{tabular}

Textura: método de Bouyoucos; MO: método de Walkley y Black; pH: relación suelo/ agua 1: 2; CIC: método de acetato de amonio neutro; Densidad Aparente: método del terrón parafinado.

Una vez reguladas las condiciones de humedad, se realizó un pulso, empleando los mismos equipos, con una solución acuosa de $\mathrm{CaCl}_{2}$ 0,01 M, que contenía 42,4 $\mathrm{mg} \mathrm{L}^{-1}$ de bromuro y 3,97 $\mathrm{mg} \mathrm{L}^{-1}$ de oxadixyl a un flujo de $5,5 \mathrm{~mL} \mathrm{~h}^{-1}$. Después del pulso se simuló una lluvia artificial manteniendo el mismo flujo con solución acuosa de $\mathrm{CaCl}_{2} 0,01 \mathrm{M}$, hasta que no se detectaron más los solutos en los percolados. El contenido de agua en el suelo fue medido con una sonda (ThetaProbe type ML2x/d, AT Delta-T Devices) conectada a un "datalogger" (HH2 Moisture Meter), localizada a la mitad de la altura de la columna. Los lixiviados fueron colectados en intervalos de 5 horas en frascos de $120 \mathrm{~mL}$ con ayuda de un automuestreador y los volúmenes fueron determinados gravimétricamente. Las muestras se filtraron con filtros de jeringa de $0,45 \mu \mathrm{m}$ y fueron almacenados a $4^{\circ} \mathrm{C}$ hasta el momento del análisis.

\subsection{Análisis de muestras de los lixiviados}

La concentración de los solutos en los lixiviados se determinó en HPLC (High Performance Liquid Chromatography), serie 1100 Agilent Technologies (Palo Alto, CA, EUA), equipado con bomba cuaternaria, inyector manual (20 $\mu \mathrm{L} \mathrm{Loop})$, y detector de arreglo de diodos (DAD) según la metodología descrita por Pot et al. (2005). La columna analítica empleada para bromuro fue una Alltech Alsep $(100 \times 4,6 \mathrm{~mm}, 7 \mu \mathrm{m})$ y como fase móvil se empleó un buffer acuoso $100 \% \quad \mathrm{NaHCO}_{3} / \mathrm{Na}_{2} \mathrm{CO}_{3}$ 2,8/2,2 nM, en modalidad isocrática, con un flujo $1,2 \mathrm{~mL} \mathrm{~min}^{-1}$. El tiempo de retención para bromuro fue de 6,1 min. Los límites de detección (LD) y cuantificación (LC) fueron $0,14 \mathrm{mg} \mathrm{L}^{-1}$ y $0,45 \mathrm{mgL}^{-1}$ respectivamente. Para la determinación de las concentraciones de oxadixyl, se empleó una columna analítica RP-18 (25 cm x $4 \mathrm{~mm}$ d.i., $5 \mu \mathrm{m}$ d.p.). La fase móvil fue acetonitrilo: agua (70:30), en modalidad isocrática, con un flujo de $0,8 \mathrm{~mL} \mathrm{~min}^{-1}$. La longitud de onda se fijó en $240 \mathrm{~nm}$. El tiempo de retención de oxadixyl fue de 3,5 min. Los límites LD y LC fueron $0,05 \mathrm{mg} \mathrm{L}^{-1}$ y $0,16 \mathrm{mg} \mathrm{L}^{-1}$ respectivamente.

\subsection{Estimación de parámetros de transporte}

En la estimación de los parámetros transporte se utilizó el programa Stanmod (Šimůnek et al., 1999) que incluye los algoritmos de CXTFIT 2.1 (Toride et al., 1999). Este programa 
presenta numerosas soluciones analíticas para modelos de transporte, basadas en la ecuación de convección-dispersión y ha sido ampliamente usado por otros investigadores (van Genuchten et al., 2012). En este caso se escogieron los siguientes modelos determinísticos de transporte: a) en condiciones de equilibrio (Convection-Dispersion Equation, CDE), b) no equilibrio físico (Physical Non Equilibrium, PNE) y c) no equilibrio químico (Chemnical Non Equilibrium, CNE). El programa se empleó en la resolución del problema inverso, mediante el ajuste de las soluciones matemáticas teóricas, a los resultados experimentales.

El valor de $v$ fue calculado a partir del valor promedio de flujo a la salida de la columna y el valor promedio del contenido volumétrico de agua $\left(\Theta=0,42 \pm 0,2 \mathrm{~cm}^{3} \mathrm{~cm}^{-3}\right)$ y fijado en los modelos matemáticos, y el coeficiente de dispersión hidrodinámica $D$, se estimó a partir de los datos experimentales obtenidos para el bromuro mediante los modelos CDE y PNE. $R$, el factor de retardo, está relacionado con la adsorción de cada soluto durante su transporte a través del suelo, y se fijó igual a 1 para bromuro (Montoya et al., 2006; Köhne et al., 2011). Con el modelo PNE, adicionalmente se evaluó que tuvieran lugar procesos de no equilibrio de origen físico, y se estimaron los valores de $\beta$ (relación entre la porosidad de la fase móvil y la porosidad total útil para el flujo de agua) y $\omega$ (coeficiente adimensional de transferencia de materia entre la fase móvil e inmóvil).

Para el fungicida, los resultados experimentales se modelaron además en condiciones de no equilibrio químico. Este modelo considera que los sitios de adsorción se dividen en dos fracciones, sitios Tipo-1 en los cuales la adsorción ocurre de manera instantánea y viene descrita por las isotermas de adsorción, y sitios Tipo-2 en los que la adsorción va a ser dependiente del tiempo y seguirá cinéticas de primer orden (Langer et al., 1999). Las ecuaciones que gobiernan este modelo tienen la misma forma adimensional que las que describen PNE, sin embargo alguno de los parámetros adimensionales no tienen el mismo significado (Van Genuchten y Wagenet, 1989).

La mejor solución al problema inverso se obtiene minimizando la función objetivo (SSQ), que es la sumatoria de los cuadrados de las diferencias entre la concentración real y la concentración ajustada por el modelo (Maquardt, 1963). El coeficiente de determinación $r^{2}$ nos da idea de la bondad del ajuste de la regresión entre la concentración observada y la calculada por el modelo y la varianza (MSE) se estima por aproximación del valor medio del error al cuadrado.

El modelo se aplicó bajo condiciones de sistema semi-infinito, inyección de pulso y cinéticas de degradación únicamente en la fase líquida. Las condiciones iniciales, las condiciones limitantes y las soluciones analíticas están detalladas en el manual del programa (Toride et al., 1999).

\section{RESULTADOS Y DISCUSION}

\subsection{Curvas de elución (BTC) de bromuro y de Oxadixyl}

Las curvas de elución se obtuvieron representando la concentración relativa del bromuro y oxadixyl en solución (cociente entre la concentración en el efluente y la concentración inicial aplicada en la solución, $\mathrm{C} / \mathrm{C}_{0}$ ) frente al volumen de elución relativo (cociente entre el volumen de elución en el tiempo y el volumen de poro, $\mathrm{V} / \mathrm{V}_{0}$ ). Para cada punto de concentración se consideró como tiempo real, el tiempo medio del intervalo de colecta, ya que la concentración final en cada fracción es la media de las concentraciones del efluente registradas en ese intervalo de tiempo.

En la Figura 1, donde se comparan las curvas de elución de bromuro y oxadixyl, se observa una temprana aparición en los lixiviados del fungicida, a tan sólo $0,7 \mathrm{~V} / \mathrm{V}_{0}$. La curva del plaguicida presenta amplia asimetría, estando desplazada hacia la derecha, con un gran 
incremento en la cola de elución o "tailing" comparada con la curva del bromuro. Esta forma de la curva de elución es indicativa de un fenómeno de transporte en condiciones de no equilibrio.

El balance de masa de bromuro dio un porcentaje de recuperación de 92,6\% y su tiempo de pico de elución fue de $0,397 \mathrm{~V} / \mathrm{V}_{0}$, resultado acorde con lo esperado, ya que bromuro es un elemento traza no reactivo (Montoya et al., 2006; Köhne et al., 2011). El porcentaje de recuperación de oxadixyl fue de $92,1 \%$, indicando su baja retención en suelo y un alto potencial de lixiviación hacia aguas subterráneas, probablemente relacionado con su alta solubilidad en agua. Las altas tasas de recuperación de la molécula indican que los mecanismos de adsorción iniciales en suelo son reversibles con el tiempo y que no hay degradación de la molécula durante el desarrollo de la infiltración. Prácticamente todo el fungicida es lixiviado del suelo después de $2,13 \mathrm{~V} / \mathrm{V}_{0}$ (cerca de 1 mes).

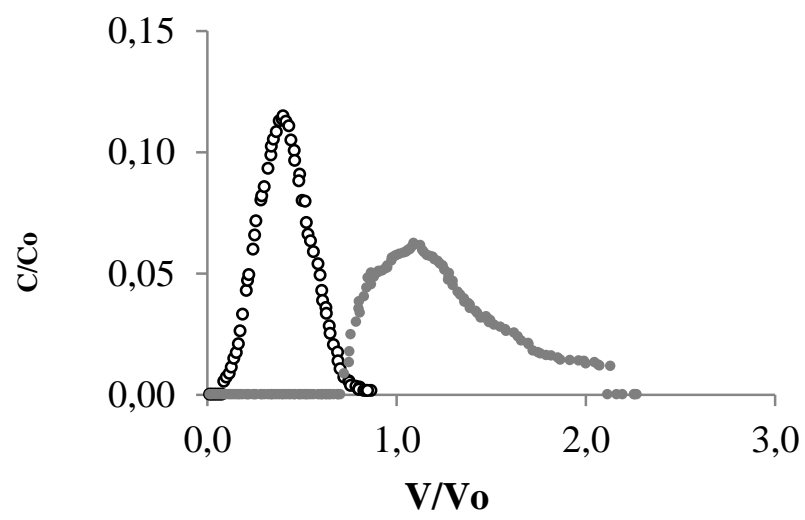

Figura 1. Comparación de las curvas de elución bromuro y oxadixyl: (o) Bromuro;

(•) Oxadixyl.

\subsection{Estimación de parámetros de transporte}

Para describir el comportamiento de Bromuro en la columna, se utilizaron los modelos CDE y PNE como se observa en la Figura 2. Gráficamente aunque los dos modelos se ajustaron al inicio de la curva, se obtuvo una mejor descripción del pico máximo de concentración con PNE (Figura 2a). Igualmente desde el punto de vista estadístico (Tabla 2), el modelo que mejor se ajustó fue PNE, ya que se encontró el mínimo valor para la función SSQ $\left(5,05 \times 10^{-3}\right)$. Los resultados muestran que en las condiciones de estudio existen dos regiones de agua en la columna: móvil e inmóvil, indicando que solo el 43,2\% del agua participa en el movimiento transversal de solutos y que la transferencia de masa entre ambas regiones es muy pequeña $(\omega=0,184)$.

A partir del coeficiente de dispersión, se calculó la dispersividad $\lambda=2,28 \mathrm{~cm}$. Este valor está dentro de los límites esperados para este tipo de suelo en función de su textura y de la profundidad (Tabla 1) (Perfect et al., 2002; Vanderborght y Vereecken, 2007).

En la Tabla 3 se muestran los resultados del ajuste de los modelos PNE y CNE para oxadixyl, empleando los valores de $v=0,157 \mathrm{~cm} \mathrm{~h}^{-1}$ (calculado a partir del valor promedio de humedad) y $D=0,36 \mathrm{~cm}^{2} \mathrm{~h}^{-1}$ (obtenido para el bromuro con el modelo PNE), teniendo en cuenta en ambos casos la posible degradación del fungicida. El modelo PNE, no se ajustó bien a los datos experimentales de oxadixyl. Los mejores valores de la función SSQ y de SME se obtuvieron en este caso para el modelo de no equilibrio químico sin degradación, aunque no describe totalmente la forma del pico de elución del oxadixyl (Figura 2b), muestra un coeficiente de correlación $\mathrm{r}^{2}$ de 0,9396 debido a que si puede describir adecuadamente al ascenso y descenso de la BTC. Cuando se introdujo el término de degradación al ajuste matemático, se obtuvo un resultado similar, pero el valor de $\mu=1 \times 10^{-9} \mathrm{~h}^{-1}$ (fijado por 
CXTFIT) es prácticamente despreciable, mostrando que no hay degradación en la fase líquida y concordando con que casi la totalidad del fungicida fue lixiviado. Debido a la alta solubilidad y polaridad del compuesto podríamos esperar una adsorción en las arcillas y MO del suelo despreciables, sin embargo los resultados de la modelación están mejor descritos por el modelo CNE. En este tipo de transporte en condiciones de no equilibrio químico, la descripción de los procesos de adsorción implicados puede ser ambigua para solutos débilmente adsorbidos, pudiendo tratarse de procesos de difusión molecular hacia el interior de los microagregados formados por MO, arcillas y otras partículas de suelo (Van Genuchten y Wierenga, 1976; Beigel y Di Pietro, 1999).
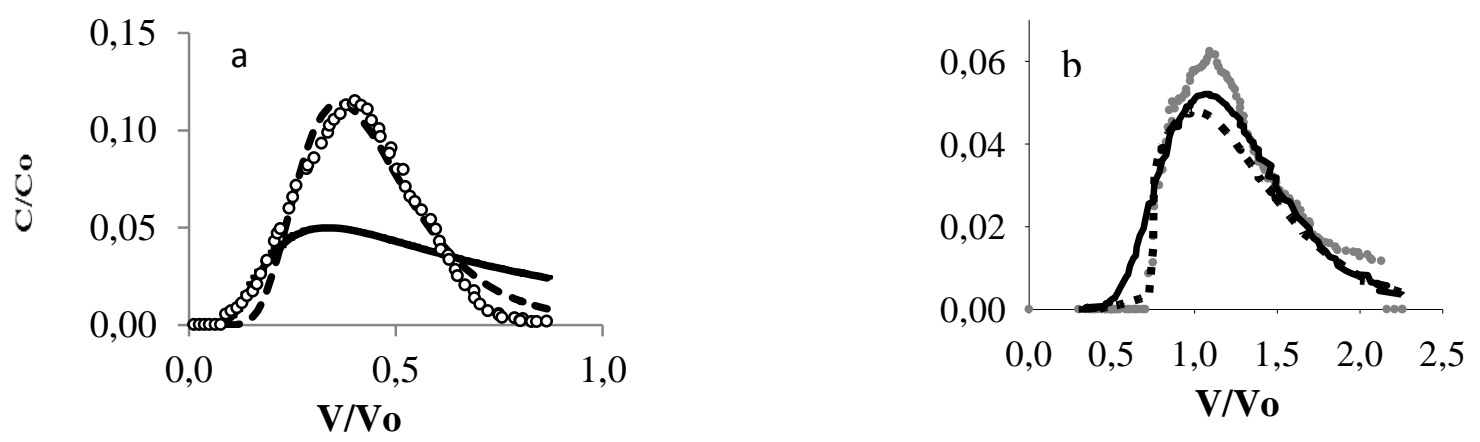

Figura 2. Comparación de las curvas de elución obtenidas experimentalmente para a) el bromuro (o) con las curvas obtenidas por modelación: (--) curva ajustada por PNE; ( - ) curva ajustada por CDE y b) oxadixyl ( $\bullet$ ) con la curva obtenida por modelación (--) curva ajustada por PNE ( $\boldsymbol{-}$ ) curva ajustada por CNE.

Tabla 2. Parámetros de transporte obtenidos para el bromuro por el modelo CDE y PNE. Entre paréntesis, debajo de cada valor, se encuentran las desviaciones estándar para cada parámetro.

\begin{tabular}{llllllllc}
\hline & $v \mathbf{c m ~ h}^{-1}$ & $\boldsymbol{D} \mathbf{~ c m}^{2} \mathbf{h}^{-1 * *}$ & $\boldsymbol{R}$ & $\boldsymbol{\beta}$ & $\boldsymbol{\omega}$ & $\boldsymbol{r}^{2}$ & SME & SSQ \\
\hline $\mathbf{C D E}$ & $0,157 *$ & $2,29 * *$ & $1 *$ & - & - & 0,367 & $1,0 \times 10^{-3}$ & $6,7 \times 10^{-2}$ \\
& $(0,025)$ & $(0,14)$ & & & & & & \\
PNE & $0,157 *$ & $0,36^{* *}$ & $1 *$ & $0,432 * *$ & $0,184 * *$ & 0,946 & $7,7 \times 10^{-5}$ & $5,05 \times 10^{-3}$ \\
& $(0,025)$ & $(0,16)$ & & $(0,113)$ & $(0,190)$ & & & \\
\hline
\end{tabular}

*Fijado ** Estimado por CXTFIT.

Ya que el modelo que mejor se ajustó en la descripción del transporte del plaguicida fue CNE, podemos deducir que el valor de la fracción de agua inmóvil encontrada en la modelación del bromuro empleando PNE, está relacionada con un proceso de exclusión aniónica del ión, ya que este suelo posee un alto contenido de arcillas (Tabla 1), más que a la existencia de regiones móviles e inmóviles de agua en la columna. Esta hipótesis estaría además confirmada por el pequeño valor de $\omega$.

El valor del coeficiente de retardo $\mathrm{R}=3,94$ de oxadixyl, ajustado al modelo CNE sin degradación (Tabla 3), muestra una demora en el transporte vertical del fungicida con respecto al elemento traza. El valor promedio de $\mathrm{Koc}=19,4 \pm 1,6 \mathrm{~L} \mathrm{~kg}^{-1}$, calculado como el cociente entre $\mathrm{Kd}$ y el porcentaje de carbono orgánico, es de orden de valores reportados en la base de datos de la Iupac (2014) y muestra la pobre afinidad del fungicida por la materia orgánica, a pesar de que el contenido de MO de estos suelos, alrededor del 5\% (Tabla 1), es 
elevado en comparación con otros estudios de lixiviación de fungicidas que mostraron un comportamiento similar (Beigel y Di Pietro, 1999; Peña et al., 2011).

Tabla 3. Comparación de los parámetros de transporte de oxadixyl obtenidos para el modelo PNE y CNE con y sin degradación. Entre paréntesis, debajo de cada valor, se encuentran las desviaciones estándar para cada parámetro.

\begin{tabular}{|c|c|c|c|c|}
\hline \multirow{2}{*}{ Parámetro } & \multicolumn{2}{|c|}{ PNE } & \multicolumn{2}{|c|}{ CNE } \\
\hline & + Degradación & - Degradación & + Degradación & - Degradación \\
\hline \multirow[t]{2}{*}{$R$} & $1,23 *$ & $1,23^{*}$ & $3,93 *$ & $3,94 *$ \\
\hline & $(0,12)$ & $(0,12)$ & $(0,22)$ & $(0,22)$ \\
\hline \multirow[t]{2}{*}{$\mathrm{Kd}\left(\mathrm{LKg}^{-1}\right)$} & $0,0816^{* * *}$ & $0,0782 * * *$ & $1,01 * * *$ & $1,00 * * *$ \\
\hline & $(0,12)$ & $(0,11)$ & $(0,23)$ & $(0,22)$ \\
\hline \multirow{2}{*}{$\boldsymbol{\beta}$} & $0,432 * *$ & $0,432 * *$ & $0,320^{*}$ & $0,315^{*}$ \\
\hline & $(0,113)$ & $(0,113)$ & $(0,118)$ & $(0,121)$ \\
\hline$\omega$ & $\begin{array}{l}0,184 * * \\
(0,190)\end{array}$ & $\begin{array}{l}0,184 * * \\
(0,190)\end{array}$ & $\begin{array}{l}1 \times 10^{-7} * \\
(0,037)\end{array}$ & $\begin{array}{l}1 \times 10^{-7} * \\
(0,037)\end{array}$ \\
\hline$\mu$ & $\begin{array}{l}0,1 \times 10^{-6 *} \\
(3,7)\end{array}$ & - & $\begin{array}{l}1 \times 10^{-9} * \\
(7,4)\end{array}$ & - \\
\hline $\mathbf{r}^{2}$ & 0,7330 & 0,7309 & 0,9380 & 0,9396 \\
\hline SME & $0,4758 \times 10^{-2}$ & $0,4738 \times 10^{-2}$ & $0,3011 \times 10^{-4}$ & $0,3237 \times 10^{-4}$ \\
\hline SSQ & $0,5753 \times 10-1$ & $0,4725 \times 10-1$ & $0,4123 \times 10-2$ & $0,4553 \times 10-2$ \\
\hline
\end{tabular}

* Estimado por CXTFIT. **Fijado *** Calculado a partir de R.

La fracción de sitios Tipo- $1, f$, calculada a partir del valor de $\beta$, nos indica que sólo el $41 \%$ de los sitios del suelo son directamente accesibles a la adsorción instantánea, siendo mayoritarios el número de sitios de más difícil acceso, por eso la adsorción es más lenta (difusión al interior de los agregados), lo que concuerda gráficamente por la extensa cola de elución de oxadixyl. El valor calculado del coeficiente de primer orden para los sitios Tipo-2, $\alpha$ es de tan sólo $7,6 \times 10^{-10} \mathrm{~h}^{-1}$, lo que corrobora una lenta adsorción de los sitios de Tipo-2 (Toride et al., 1999).

\section{CONCLUSIONES}

En este estudio se muestra que oxadixyl tuvo una alta movilidad en las columnas de suelo, no presentándose fenómenos de degradación. El programa Stanmod permitió caracterizar el transporte del bromuro en condiciones de no equilibrio de tipo físico indicando que en la columna existen dos regiones de agua, móvil e inmóvil. Sin embargo el pequeño valor del coeficiente de difusión de masa entre las dos regiones es muy pequeño, mostrando que el en realidad no se trata de un proceso de no equilibrio físico en la columna sino de un fenómeno de repulsión entre las cargas negativas del suelo (de las arcillas) y el elemento traza.

Para oxadixyl se encontró que el modelo que mejor describía el movimiento a través del perfil del suelo era el de no equilibrio de tipo químico, aunque los parámetros obtenidos con PNE y el elevado porcentaje de recuperación, superior al 92\%, sugieren que se trata de una retención débil en suelo, probablemente debida a la difusión lenta de la molécula al interior de los agregados del suelo, que provoca un retraso (pero no impedimento de su transporte) hacia las aguas subterráneas.

Debido a que los resultados obtenidos son limitados a un solo ensayo, es necesario realizar mayor número de estudios tanto en condiciones de campo como de laboratorio para 
información más precisa y concluyente sobre el riesgo de contaminación de las aguas del Lago de Tota.

\section{AGRADECIMIENTOS}

Los autores agradecen al proyecto Código 7553 de la Dirección de Investigación Sede Bogotá, de la Universidad Nacional de Colombia por el apoyo económico para el desarrollo de este trabajo.

\section{REFERÊNCIAS}

ABELLA, J. P.; MARTÍNEZ, M. J. Contribución de un afluente tributario a la eutrofización del Lago de Tota (Boyacá, Colombia). Revista Colombiana de Química, v. 41, n.1, p. 243-261, 2012.

BEIGEL, C.; DI PIETRO, L. Transport of triticonazole in homogeneous soil columns: influence of nonequilibrium sorption. Soil Science Society of America Journal, v. 63 n. 5, p. 1077-1086, 1999. http://dx.doi.org/10.2136/sssaj1999.6351077x

BLANCHOUD, H.; MOREAU-GUIGON, E.; FARRUGIA, F.; CHEVREUIL, M.; MOUCHEL, J. M. Contribution by urban and agricultural pesticide uses to water contamination at the scale of the Marne watershed. Science of the Total Environment, v. 375, n. 1/3, p. 168-179, 2007. http://dx.doi.org/10.1016/j.scitotenv.2006.12.009

HERRERA, G.; SÁNCHEZ, V.; PEÑA, C. Avances de resultados de investigación en cebolla de rama en Aquitania, Boyacá. Bogotá: CORPOICA;CORPOBOYACA, 2006. 2740 p.

INSTITUTO COLOMBIANO AGROPECUARIO - ICA. Listado de Registros nacionales de plaguicidas químicos de uso agrícola. Disponible en: http://www.ica.gov.co/Areas/Agricola/Servicios/Regulacion-y-Control-dePlaguicidasquimicos.aspx. Acceso: 10 Septiembre 2014.

INTERNATIONAL UNION OF PURE AND APPLIED CHEMISTRY - IUPAC. Pesticide properties database. Disponible en: http://agrochemicals.iupac.org. Acceso: 4 Noviembre 2014.

KÖHNE, J. M.; SCHLÜTER, S.; VOGEL, H. J. Predicting solute transport in structured soil using pore network models. Vadose Zone Journal, v. 10, n. 3, p. 1082-1096, 2011. http://dx.doi.org/10.2136/vzj2010.0158

LANGER, H. W.; GABER, H. M.; WRAITH, H. M.; HUWE, B.; INSKEEP, W. P. Preferential flow through intact soil cores: effects of matrix head. Soil Science Society of America Journal, v. 63, p. 1591-1598, 1999.

http://dx.doi.org/10.2136/sssaj1999.6361591x

MARQUARDT, D. W. An algorithm for least-squares estimation of nonlinear parameters. Journal of the Society for Industrial, v. 11, p. 431-441, 1963. http://dx.doi.org/10.1137/0111030

MÓJICA, A. Determinación de residuos de plaguicidas en aguas y suelos de la región del Lago de Tota-municipio de Aquitania. 2010. 151f. Tesis (Maestría) - Universidad Nacional de Colombia, Bogotá, 2010. 
MOLINA, A. Cuenca hidrográfica del lago de Tota. Bogotá: Taller de Cooperación Técnica AIEA, 2007.

MONTOYA, J. C.; COSTA, J. L.; LIEDL, R.; BEDMAR, F.; DANIEL, P. Effects of soil type and tillage practice on atrazine transport through intact soil cores. Geoderma, v. 137, p. 161-173, 2006. http://dx.doi.org/10.1016/j.geoderma.2006.08.007

PEÑA, A.; PALMA, R.; MINGORANCE, M. D. Transport of dimethoate through a Mediterranean soil under flowing surfactantsolutions and treated wastewater. Colloids and Surfaces A: Physicochemical and Engineering Aspects, v. 384, p. 507- 512, 2011. http://dx.doi.org/10.1016/j.colsurfa.2011.05.024

PERFECT, E.; SUKOP, M. C.; HASZLER, G. R. Prediction of dispersivity for undisturbed soil columns from water retention parameters. Soil Science Society of America Journal, v. 6, p. 696-701, 2002. http://dx.doi.org/10.2136/sssaj2002.6960

PINZÓN, H. Validación participativa y transferencia de tecnología en cebolla de rama, cebolla de bulbo $\mathbf{y}$ ajo para Cundinamarca y Boyacá. Informe final para Asohofrucol. Bogotá: Produmedios, 2004. 40 p.

POT, V.; ŠIMŮNEK, J.; BENOIT, P.; COQUET, Y.; YRA, A; MARTINEZ-CORDON, M. J. Impact of rainfall intensity on the transport of two herbicides in undisturbed grassed filter strip soil cores. Journal of Contaminant. Hydrology, v. 81, n.1-4, p. 63-88, 2005. http://dx.doi.org/10.1016/j.jconhyd.2005.06.013

SAIANO F.; CIOFALO, M. Removal of pesticide oxadixyl from an aqueous solution. Bioremediation Journal, v. 11, p.57-60, 2007. http://dx.doi.org/10.1080/10889860701351555

ŠIMU゚NEK, J.; VAN GENUCHTEN, M. T. H.; ŠEJNA, M.; TORIDE, N.; LEIJ, F. J. The STANMOD computer software for evaluating solute transport in porous media using analytical solutions of convection-dispersion equation. Versions 1.0 and 2.0. IGWMC-TPS-71. Int. Ground Water Modeling Ctr. Golden: Colorado School of Mines, 1999.

TORIDE, N.; LEIJ, F. J.; VAN GENUCHTEN, M. T. H. The CXTFIT Code for Estimating transport parameters from laboratory or field tracer experiments. Version 2.1, Research Report No. 137, Riverside: U.S. Salinity Laboratory; Agricultural Research Service, 1999.

VAN GENUCHTEN, M. T. H.; ŠIMU゚NEK, J.; LEIJ, F. J.; TORIDE, N.; ŠEJNA, M. Stanmod: model use, calibration and validation. American Society of Agricultural and Biological Engineers, v. 55, n. 4, p. 1353-1366, 2012.

VAN GENUCHTEN, M. T. H.; WAGENET, R. J. Two Site/Two-Region models for pesticide transport and degradation: theoretical development and analytical solutions. Soil Science Society of America, v. 53, p. 1303-1310, 1989. http://dx.doi.org/10.2136/sssaj1989.03615995005300050001x

VAN GENUCHTEN, M. T. H.; WIERENGA, P. J. Mass transfer studies in sorbing porous media: 1. Analytical solutions. Soil Science Society of America Journal, v. 40, p. 473480, 1976. http://dx.doi.org/10.2136/sssaj1976.03615995004000040011x 
VANDERBORGHT, J.; VEREECKEN, H. Review of dispersivities for transport modelling in soils. Vadose Zone Journal, v. 6, p. 29-52, 2007.

http://dx.doi.org/10.2136/vzj2006.0096 